\title{
GAMIFICACIÓN Y EVALUACIÓN FORMATIVA EN LA ASIGNATURA DE MATEMÁTICA A TRAVÉS DE HERRAMIENTA WEB 2.0
}

\section{GAMIFICATION AND FORMATIVE ASSESSMENT IN THE SUBJECT OF MATHEMATICS THROUGH A WEB 2.0 TOOL}

\section{Raúl Prada Núñez ${ }^{1}$}

César Augusto Hernández Suárez²

William Rodrigo Avendaño Castro ${ }^{3}$

UFPS

\section{RESUMEN}

El estudio pretendió comprender la gamificación, la evaluación formativa en una herramienta

1 Magister en Ingeniería de Análisis de Datos, Mejora de Procesos y Toma de Decisiones por la Universidad Politécnica de Valencia (España). Docente investigador de la Universidad Francisco de Paula Santander. Correo electrónico: raulprada@ufps.edu.co. Orcid: 0000-0001-61451786.

2 Magister en Educación Matemática por la Universidad Nacional Experimental del Táchira (Venezuela). Docente investigador de la Universidad Francisco de Paula Santander. Correo electrónico: cesaraugusto@ufps.edu.co. Orcid: 0000-0002-7974-5560.

3 Doctor en Ciencias Sociales y Humanas por la Pontificia Universidad Javeriana. Docente investigador de la Universidad Francisco de Paula Santander. Correo electrónico: williamavendano@ufps.edu.co. Orcid: 0000-00027510-8222. web2.0, y su mejora en la práctica educativa de las matemáticas en un contexto digital. La investigación se contextualizó dentro del enfoque cualitativo, el tipo fue investigaciónacción. La muestra estuvo conformada por 30 estudiantes y un docente de una Institución Educativa ubicada en la ciudad de Cúcuta, Colombia. Los instrumentos empleados fueron un cuestionario en escala Likert de cinco alternativas para evaluar la percepción de los estudiantes en relación a la estrategia de gamificación; informes de evaluación formativa y las retroalimentaciones. También, se utilizó la observación para datos cualitativos. Se halló que 
la evaluación formativa en una herramienta Web de la asignatura de matemática en un ambiente no lúdico y gamificado depende de los métodos de realimentación utilizados por el docente en dar respuestas a las inquietudes y solicitudes presentadas por los estudiantes. Se concluyó que cuanto más aumenta la gamificación en sus elementos y la evaluación formativa en el contexto digital más cambia significativamente el aprendizaje de las matemáticas.

\section{PALABRAS CLAVES:}

evaluación formativa, Gamificación, herramienta web2.0, matemática.

\section{ABSTRACT}

The study aimed to understand gamification, formative evaluation in a web2.0 tool, and its improvement in the educational practice of mathematics in a digital context. The research was contextualized within the qualitative approach, the type was action research. The sample consisted of 30 students and a teacher from an Educational Institution located in the city of Cúcuta, Colombia. The instruments used were a questionnaire on a Likert scale of five alternatives to evaluate the perception of the students in relation to the gamification strategy; formative evaluation reports and feedbacks. Also, observation was used for qualitative data. It was found that the formative evaluation in a Web tool of the mathematics subject in a nonplayful and gamified environment depends on the feedback methods used by the teacher in responding to the concerns and requests presented by the students. It was concluded that the more gamification increases in its elements and the formative evaluation in the digital context, the more significantly the learning of mathematics changes.

\section{KEYWORDS:}

formative assessment, Gamification, web2.0 tool, mathematics.

\section{INTRODUCCIÓN}

La educación del siglo XXI se ha circunscrito en enseñar a los estudiantes las habilidades que necesitan para triunfar en la generación del conocimiento del mundo globalizado (Villanueva y Casas, 2010). Las directrices del Ministerio de Educación Nacional (MEN) promueve nivel educativo por competencias en pensamiento crítico, trabajo colaborativo, en TIC en el manejo de la información y ser matemáticamente competente, entre otras, dado que, "la educación actual requiere de personas con capacidad crítica, analítica, reflexiva... con un desarrollo intelectual alto... para interpretar, argumentar, proponer, plantear y resolver problemas en diferentes contextos" (Aristizábal, Colorado Gutiérrez, 2016, p. 119).

Por otra parte, la aplicación las TIC a través de equipos y dispositivos en el aula, ambientes virtuales de aprendizaje, evaluación electrónica y uso de redes sociales de acuerdo con Rojas, Beleño y Valbuena (2015) eleva el aprendizaje y apoya la enseñanza de la matemática. Aunque estos autores, argumentan que requieren de acceso técnico, práctico y operativo, así como equidad, currículo, enseñanza, aprendizaje, evaluación y tecnología.

A través de nuevas tendencias educativas pareciera que se unen cada día más las TIC, las matemáticas y la evaluación. Las tecnologías no solo han traído consigo avances, sino también nos han sumergido en profundos procesos de cambio para tratar de adaptarse al reto que plantea este nuevo escenario (Martínez, 2017). Donde se han incluido el "aprendizaje basado en juegos y la gamificación como estrategia didáctica, integrando aspectos de la dinámica del juego en contextos no lúdicos que ayuden a potenciar la motivación de los estudiantes..." (Ortiz-Colón, Jordán y Agredal, 2018, p.3). 
Para lograr estos objetivos, el punto de vista principal no está solo en la calificación sino en la evaluación formativa que apoya el desarrollo del estudiante por su potencial para mejorar el aprendizaje (Martínez Rizo, 2012; García Quintero y Villamizar Suárez, 2017), a través, de la control, recopilación, análisis y presentación de datos de los estudiantes en sus contextos digitales o físico.

A nuestro entender, las tendencias e inquietudes actuales exigen análisis de aprendizaje y del conocimiento de acuerdo con la Society for Learning Analytics Research (2010, citado en Siemens y Baker, 2012). Porque cada día las generaciones de estudiantes necesitan encontrar respuesta en el contexto educativo a sus expectaciones tecnológicas y necesidades más inmediatas., es decir, una contestación ludificada a los juegos, su organización individualizada y colectiva de la actividad estimulada por la tarea a través del aprendizaje experiencial y por descubrimiento.

La solución parece sencilla, hace que el entorno de aprendizaje y el trabajo sean lúdicos, donde las actividades que requieren esfuerzo se vuelven atractivas y gratificantes para los participantes, pues pasan tiempo en dichas actividades por el simple placer de hacerlas. (Csikszentmihalyi, 1990). Pero trabajos recientes demuestran que la evaluación gamificada es valorada por los docentes (Colomo, Sánchez, Ruiz y Sánchez, 2020), por dar continuidad al aprendizaje fuera del contexto escolar.

De allí, que la gamificación es de gran interés investigativo en la educación (Dicheva, Dichev, Agre \& Angelova, 2015; Ortiz-Colón, Jordán y Agredal, 2018), De hecho, las Tecnologías y nuevas tendencias en educación se orientan para aprender jugando (Martínez, 2017; LópezGarcía y Gutiérrez-Niño, 2018) y la gamificación a través de una plataforma para mejorar el rendimiento académico en matemáticas
(Sánchez, 2018). Es posible, que los juegos estén conducidos de una creencia optimista en las habilidades de las personas y la voluntad de actuar que lo acompañan.

También, la gamificación fue utilizada como estrategia didáctica que apoyó el desarrollo de habilidades en el planteamiento y resolución de problemas aritméticos (Casallas y Maheca, 2019, así como en la enseñanza de las matemáticas (Holguín; Holguín y García, 2020). De igual manera, como elemento motivador y colaboración para equipos (Castro, 2016). También, el impacto en el escenario educativo (Erenli, 2013) y sus posibilidades en el entorno de blended learning (Torres, Romero y Pérez, 2017) como herramienta para involucrar el aprendizaje de los estudiantes con una aplicación gamificada (Welbers et al. 2017), y especialmente pensada para disfrutar de retos en las matemáticas mediante juegos interactivos en una web gratuita (retomates) (Perea, 2016).

Las exploraciones anteriores sobre este tema exponen aspectos importantes de la gamificación en diferentes áreas del conocimiento y destacan que las prácticas gamificadas se convierten en mejoras en los resultados de aprendizaje. Sin embargo, pocos o ninguno ha indagado sobre la gamificación de la evaluación en la asignatura de matemática. Además, la incorporación de estrategias de gamificación se está tornando dinámicas porque la técnica se aleja de la memorización enfocándose en la comprensión de las clases a través de un entorno interactivo y emocionante (Holguín, Holguín y García, 2020).

Por tanto, es fundamental sistematizar la información disponible sobre qué elementos se están concentrando y examinar cómo se incorporan a la evaluación formativa para aprender, pues la misma, se lleva a cabo, a partir de la observación y análisis de los datos, ya sea del proceso de aprendizaje con el fin de tomar decisiones orientadas a mejorar el trabajo 
y ayudar a progresar en la evaluación para el aprendizaje (Fernández, 2017; Penagos, Mariño y Hernández, 2017).

Independientemente, de los estudios que utilizan la gamificación en diferentes áreas de conocimiento, seconoce pocosobre la evaluación formativa y gamificación en una herramienta web. Por consiguiente, es importante entender el fenómeno en estudio. En consecuencia, el objetivo investigación es comprender la gamificación, la evaluación formativa en una herramienta web2.0, y su mejora en la práctica educativa de las matemáticas en un contexto digital

\section{BASE TEÓRICA}

\subsection{Gamificación}

La gamificación tiene su fundamento en el diseño de juegos. Un juego de acuerdo con Salen y Zimmerman (2004, p.93) "es un sistema en el que los jugadores participan en un conflicto artificial, que se define por reglas y que se traduce en un resultado cuantificable". Por otro lado, la gamificación aplicada a la educación según Vilches (2014, p.25) "consiste en emplear mecánicas de juego en entornos o ambientes no lúdicos".

La gamificación como estrategia didáctica cumple con seis etapas: "1) define los objetivos de aprendizaje; 2) delimita las conductas que se desea lograr; 3) describe a los participantes; 4) diseña ciclos de actividades; 5) incluye diversión; 6) implementa herramientas adecuadas" (Werbach y Hunter, 2012 p. 86). Asimismo, los elementos de la gamificación de acuerdo con Werbach y Hunter (ob. cit) se encuentran bajo tres categorías: dinámicas, mecánicas y componentes.

Donde la dinámica, establece las acciones de los estudiantes y están relacionadas con la motivación, ya que mezclan emociones con la progresión de las actividades y las relaciones que se originan en la misma. Mientras que la mecánica, hace referencia a los componentes básicos del juego, sus reglas y su funcionamiento.

En esta categoría, la mecánica describe los retos, competición, cooperación, recompensas y realimentación. Entendiendo que, la realimentación es esencial para el contenido de la gamificación en la educación, puesto que, permite crear una dinámica del juego enfocada en la evaluación formativa del aprendizaje (Ropero, 2018), encaminando las metas de aprendizaje mientras se proporciona acotaciones explícitas para articular los retos, la competencia y las recompensas con el aprendizaje. Entretanto que, los componentes, representa a los recursos con que se cuenta y las herramientas que se utilizaran para diseñar las actividades en la práctica de la gamificación (niveles, avatares, logros, puntuación, insignias y ranking).

Ortegón (2016) señala que los sistemas de recompensa aplicada en las matemáticas, como los puntos y las insignias pueden aumentar el compromiso de los jugadores, ya que, al asumir los retos, el participante se siente involucrado para ser reconocidos por sus logros y ranking, donde, además reciben realimentación de su proceso. En ese sentido, estos componentes se correlacionan con el escenario dinámico conductual bajo las normas del juego y sus actividades incluye la motivación (Dicheva, Dichev, Agre y Angelova, 2015). Además, el jugador (estudiante) de forma activa, resuelve en un entorno auténtico "las mecánicas del juego, ... produce acción, promueve el aprendizaje al resolver problemas" (Kapp, 2012, p.10).

\subsection{Herramienta web.20, el caso de Retomates}

Las herramientas web 2.0 responden a criterios como compartir información y situar al usuario en el centro. Las herramientas web 
2.0 abren espacios para el desarrollo de redes y permiten a los usuarios de la Web pertenecer a comunidades, lo cuales tienen como objetivo estimular una actividad en particular de sus miembros. En el caso particular de retomates, de acuerdo con Perea (2016) es una herramienta o aplicación web gratuita especialmente pensada para disfrutar de las matemáticas.

La cual está conformada por una zona para estudiante, donde aprendan matemáticas por la mecánica propia de los juegos que concentran todos los juegos interactivos matemáticos, las tareas y los campeonatos que el profesorado haya propuesto. Mientras que, la zona de profes, es pensada para la gestión de grupos, pruebas escritas en la que se pueden generar fichas, actividades, exámenes, test, entre otras actividades.

Esta superposición de zonas, genera un contexto de diferentes actividades de aprendizaje y de enseñanza con el fin de crear una nueva experiencia, particularmente con respecto al sitio Web Retomates, se podría decir que usa el principio de un mashup (Cobo y Pardo, 2007), pues agrega contenidos de otras instituciones educativas y profesores del todo el mundo para crear nuevas actividades en el sitio.

Así pues, la gestión de grupos permite asignar a un grupo de alumnos tareas y campeonatos para que practiquen distintos temas del currículo. Lo cual podría ser beneficio para aplicar la evaluación formativa junto a la gamificación en la asignatura de matemática, dado que esta herramienta Web llevan asociada un sistema de insignias que permite al alumno plantearse unos objetivos y comprobar la evolución de la consecución de éstos, al tiempo que juega y aprende matemáticas.

\subsection{Evaluación formativa}

Sobre las bases del constructivismo, Rodríguez y Ibarra (2011) destacan que la evaluación parte de la "comprensión de los estudiantes, ... retroalimentación entre iguales y la autoevaluación" (p.27), facilitado por un “...proceso permanente y objetivo para valorar el nivel de desempeño de los estudiantes en relación con el desarrollo de las competencias" (Ministerio de Educación Nacional, MEN, 2009). También, la evaluación ha sido categorizada en diagnóstico, formativa y sumativa (Brenes, 2006).

La diagnóstica, refiere comprobar hasta qué punto se han conseguido los objetivos propuestos, determina el grado de identificación o discrepancia entre éstos y los resultados. Mientras que la sumativa, pretende valorar un programa/sujeto con la intención de estimar su logro/mérito. En el caso de la formativa, la intención es ayudar al desarrollo de un programa/sujeto.

En Colombia, un estudiante debe ser evaluado formativamente de manera integral en todos los aspectos académicos, personales y sociales con criterios, procedimientos e instrumentos con el fin de recibir oportunamente respuestas a las inquietudes y solicitudes presentadas para superar sus debilidades en el aprendizaje. (MEN, 2009). De allí, se considera que lo formativo es un proceso holístico que apoya el aprendizaje, pues coadyuva al desarrollo del estudiante en correspondencia con las observancias del proceso de formación del individuo de manera continua sin evaluar el desarrollo y los logros (Pérez, Enrique, Carbó \& González, 2017).

En un contexto digital, desde la posición de Ferguson, Brasher, Clow, Cooper, Hillaire, Mittelmeier, Rienties, Ullmann, y Vuorikari, R. (2016) podría alinear las analíticas de aprendizaje con los la información y datos que dejan los estudiantes en dicho contexto (Lleixà, Gros, Mauri \& Medina, 2018). Lo que le permite al docente acomodar el currículo, los objetivos iniciales y otorgan incluso, la posibilidad de 
ajustar el proceso progresivamente de las evaluaciones mediante la personalización, la diferenciación y la individualización (Santiago y Bergmann, 2018, p.161).

Concretamente, estos últimos autores argumentan que en la evaluación formativa se debe hacer una individualización para acomodar la instrucción a las necesidades de aprendizaje de los diferentes estudiantes. Seguidamente, adaptarse a las preferencias de aprendizaje de dichos educandos (diferenciación) y luego se realiza una personalización, que refiere que la educación debe acondicionarse a las metas de aprendizaje de acuerdo con las preferencias (diferenciación) y los intereses específicos (individualización) de los diferentes estudiantes, ya que toda evaluación en el que las TIC son utilizadas se puede hacer un registro de las respuestas. (Rodríguez \& Ibarra, 2011).

2.4. Aplicación de la evaluación formativa para gamificar el aprendizaje de matemáticas en una herramienta Web

En una las propuestas pedagógicas con el uso de las TIC de la asignatura de matemática, de acuerdo con Castillo (2008) la evaluación tiene que apoyar las nociones de matemática y suministrar información útil tanto a los docentes como a los estudiantes. En un contexto digital que ha sido gamificado, los docentes según Santiago y Bergmann, (2018) entiende que debe proporcionar a los estudiantes formas diferentes de evaluación formativa, para ello, arbitra un sistema de retos con juegos interactivos. En este particular, (Mora, 2003) advierte que en la resolución de problemas y las aplicaciones matemáticas, exige una evaluación formativa permanente, incluso, deben participar también activamente los demás integrantes del proceso.

En la herramienta Web gamificada (Retomates) destinada para la enseñanza y el aprendizaje de la matemática, Perea (2016) destaca el papel activo del educando que llevan asociado un sistema de insignias, retos y niveles que permite al discente plantearse unos objetivos y comprobar la evolución de la consecución de éstos, al tiempo que juega y aprende matemáticas.

En este sentido, coexiste una experiencia personal que conlleva pensamiento, acción, descubrimiento en la que se pudieran utilizar técnicas de evaluación formativa "informal, semiformal y formal" (González, Hernández y Hernández, 2007, p.128), ya que en la primera los estudiantes sienten que no están siendo evaluados, dado que se da una observación de las actividades realizadas por ellos.

Mientras que, en la técnica semiformal, requiere que los estudiantes realicen ejercicios y prácticas y; la formal, son las pruebas o exámenes tipo test, lo cual se adapta a la lista de torneos (retos, batallas ) y tests que los docentes proponen para sus grupos en la Web gamificada de retomates y que se pudiera apoyar en la sección de estadísticas de dicha Web para consultar las puntuaciones por los estudiantes, en la generación de conocimiento en la experiencia compartida (Araya, Alfaro y Andonegui, 2007).

Por tanto, tiene sentido ajustar los esfuerzos de gamificación en un contexto digital mientras los estudiantes practican ejercicios, ya que, en una herramienta tecnológica, serviría como un portafolio de aprendizaje y, por tanto, como instrumento para la evaluación formativa. Lo que permite a los estudiantes analizar su propio aprendizaje, y en los docentes, adquisición de información, datos y resultados de aprendizaje planificados al suscitar el uso de herramientas orientada precisamente a mejorar ese compromiso (Holguin, Holguin y García, 2020), ya que el entorno lúdico anima a los estudiantes a buscar problemas, hacer preguntas $y$ responderlos, proporcionan oportunidades para el pensamiento creativo y la resolución de problemas (Sánchez, 2018). 


\section{EI MÉTODO}

\subsection{Enfoque y diseño de la investigación}

La investigación se encuadró en el enfoque cualitativo. El proyecto corresponde a un proceso acerca de la gamificación y la evaluación formativa en la asignatura de matemática dentro de una herramienta Web gamificada, con el fin de optimizar y ampliar la transformación continúa de la práctica educativa (Restrepo, 2004). El tipo fue investigación-acción (Martínez, 2004), pues la evaluación formativa del docente en el aula es un agente clave en la interpretación de las problemáticas que se presentan en el proceso de enseñanza aprendizaje.

\subsection{Contexto de la investigación}

La investigación se desarrolló en la Institución Educativa ubicada en la ciudad de Cúcuta, Colombia. Los estudiantes son del grado once y están integrados por estuvo compuesta por 61 estudiantes y un docente, dividido de la siguiente manera: 30 del curso A y 31 del Curso B. Las edades oscilan entre 15 a 17 años, de los cuales 25 son de sexo masculino y 36 femeninos. Se selecciona como sujetos de estudio a los 30 estudiantes del curso A. Por contar con la participación voluntaria de los mismo.

\subsection{Procedimiento}

Se utilizó el procedimiento donde se tienen en cuenta los aspectos más importantes de cada etapa de la investigación acción propuesto por Martínez (2004) y, sobre todo, en aquellos que tienen más relación con la situación particular o sólo lo que le interesa del objeto de estudio. La ejecución de las etapas describe la identificación del problema y su respectivo análisis, seguidamente, se formulan hipótesis, se recolecta la información necesaria y luego se categoriza la información para su estructuración, para después diseñar, ejecutar y evaluar un plan de acción, en esta última etapa, se evalúa los resultados del plan de acción, una vez ejecutados, se verifica si solucionaron el problema o no. Para el análisis de los datos se utilizó el programa de procesamiento de datos IBM SPSS Statistics v25. Los resultados se revisaron mediante estadística descriptiva y se utilizó la prueba ANOVA para comparar las medias.

\subsubsection{Formulación de hipótesis}

El análisis del problema de acuerdo con Martínez (2004) presenta un abanico de posibilidades tentativas en la cual se tiene más probabilidad de explicar y solucionar el problema a través de las hipótesis, hay que concentrar el estudio con la mejor de ellas. En ese sentido, se presenta la siguiente hipótesis:

- Hipótesis Nula: La realimentación a las inquietudes y solicitudes presentadas por los estudiantes en una herramienta web no contribuye alcanzar los niveles adecuados en la asignatura de matemática en un contexto gamificado.

- Hipótesis Alternativa: Brindar oportunamente segmentos frecuentes de realimentación a las inquietudes y solicitudes presentadas por los estudiantes en una herramienta web contribuye alcanzar los niveles adecuados en la asignatura de matemática en un contexto gamificado.

\subsubsection{Recolección de la información necesaria}

De acuerdo con Creswell (2012, p. 577), la investigación acción utiliza “...datos de tipo cuantitativo, cualitativo o de ambos, ... al centrarse en la solución de un problema específico y práctico". En este sentido, se aplicó un cuestionario en escala Likert para obtener datos cuantitativos, para evaluar la percepción de los estudiantes en relación a la estrategia de gamificación; los informes de evaluación de control o formativa y las retroalimentaciones recibidas que inciden en la motivación de los a estudiantes. De igual manera, se utilizó la observación para la recolección de los datos cualitativos. 


\subsubsection{Diseño y ejecución de un plan de acción}

\subsubsection{Identificación y análisis del} problema.

Los estudiantes se concentraron en una herramienta Web2.0 denominada Retomates, a través de la gestión de grupo para profesores. El trabajo se desarrolló mediante elementos de la gamificación a partir del modelo de Werbach y Hunter (2012), en la cual se aplicó un proceso de evaluación de control en dos ciclos diferentes de realimentación inmediatamente después de realizar una actividad, prueba, o una vez que se ha proporcionado batalla con otro compañeros de acuerdo con actividad gamificada, que consistió en resolver tres desafíos con niveles de dificultad progresivo y competencia de resolución de problemas matemáticos; se manejaron avatares y un tablero para el registro de insignias dentro de la plataforma basada en recompensa.

De allí, se requiere comprender la información recibida por el estudiante (comentarios y observaciones por parte del docente) luego de realizadas las actividades gamificada en la herramienta Retomates. Es decir, qué tipo de realimentación se percibe como más útil para profesores y estudiantes que pueden mejorar la evaluación formativa en una herramienta web2.0, por ello, se examina la tecnología de juegos en relación con (la realimentación) componentes de la evaluación formativa en la asignatura de matemática en un contexto digital

En particular, se constató que los estudiantes necesitan apoyo para comprender la asignatura de matemática, así como cómo navegar e interactuar con el entorno gráfico. La falta de familiaridad de conceptos básicos y controles de navegación puede influir negativamente en la práctica y motivación de los estudiantes, pero se considera, que la realimentación dentro de la gamificación puede apoyar este proceso de familiarización. En ese sentido, La realimentación es clave componente de evaluaciones formativas, a través de comentarios con el fin de mejorar aprendizaje de los estudiantes, pues, media en la motivación y la calidad durante su interacción con el sistema de evaluación en una herramienta web gamificada en la asignatura de matemática.

Se presentan las categorías iniciales de acuerdo al problema y los objetivos de investigación.

Tabla 1. Categorías

\begin{tabular}{|l|l|}
\hline Categorías & \multicolumn{1}{c|}{ Definición } \\
\hline Motivación & $\begin{array}{l}\text { Examina la capacidad innovadora y reflexión de los } \\
\text { estudiantes. }\end{array}$ \\
\hline Gamificación & $\begin{array}{l}\text { Comprender dinámica, mecánicas y componentes de } \\
\text { juego en entornos o ambientes no lúdicos. }\end{array}$ \\
\hline Evaluación formativa & $\begin{array}{l}\text { Proceso holístico de la evaluación coadyuva al desarrollo } \\
\text { del estudiante en correspondencia con las observancias } \\
\text { del proceso de formación del individuo y con las } \\
\text { finalidades sociales que signan dicha formación en la } \\
\text { sociedad. }\end{array}$ \\
\hline realimentación & $\begin{array}{l}\text { Información diagnóstica comunicada para ayudar al } \\
\text { estudiante a saber qué y cómo mejorar en su aprendizaje. }\end{array}$ \\
\hline
\end{tabular}




\section{RESULTADOS}

\subsection{Percepciones de los estudiantes sobre motivación y elementos de gamificación en ambiente no lúdico}

Tabla 2. Motivación y elementos de gamificación en ambiente no lúdico

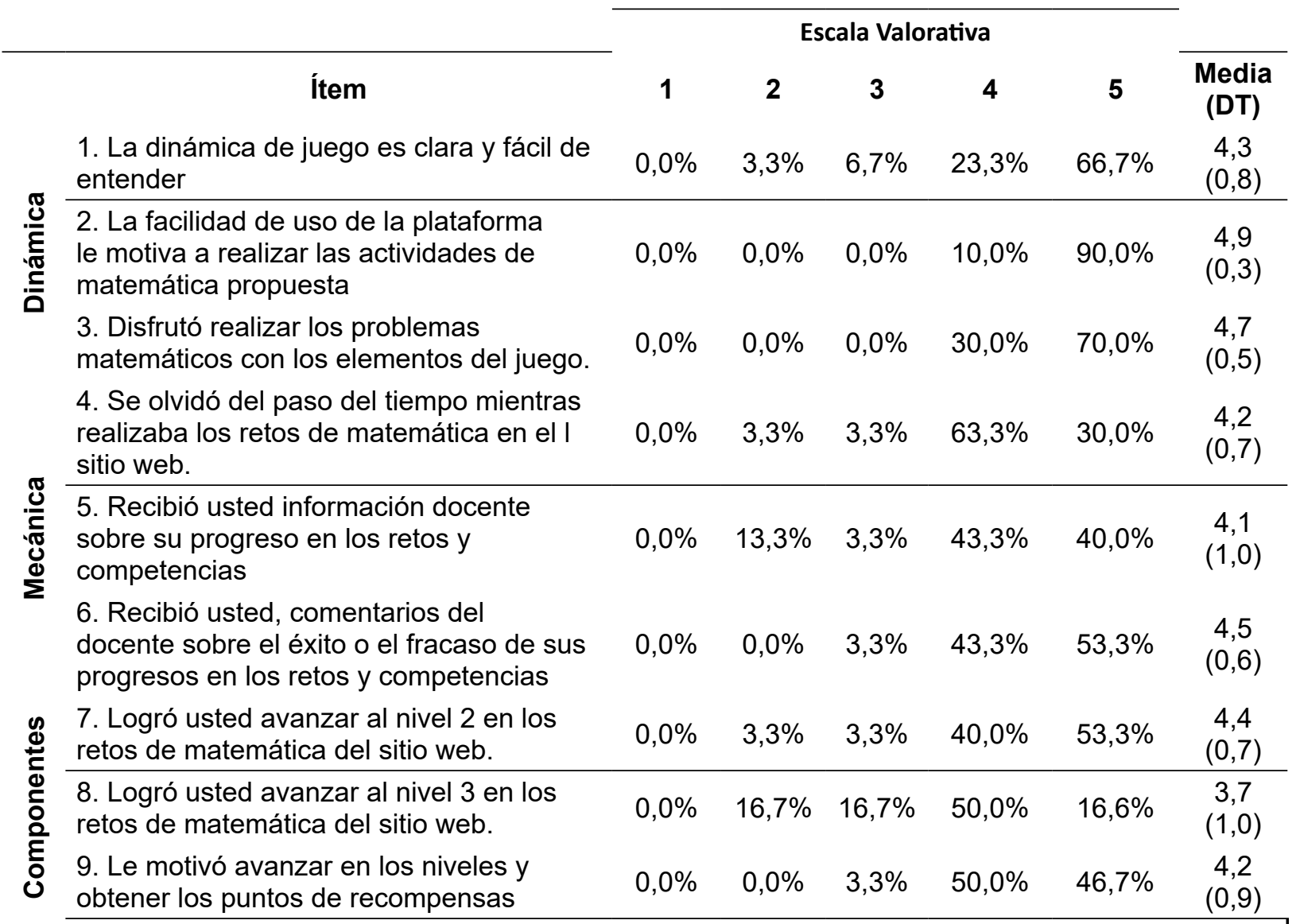

Escala evaluativa: 1. Totalmente en desacuerdo; 2. En desacuerdo; 3. Indiferente; 4. De acuerdo; 5. Totalmente de acuerdo

Se muestran los principales resultados sobre los elementos de gamificación, en la tabla 2, se halló en las actuaciones de los estudiantes un nivel de progreso esperado $(93,0 \%$ y $66,7 \%$ respectivamente) en los componentes gamificado del sitio web de Matemática. En dicha subcategoría: componentes del juego. Los estudiantes lograron niveles óptimos por la facilidad de uso de la plataforma $(100,0 \%)$ por ser claro y comprensibles $(90,0 \%)$ para entender la actividad, prueba, o una vez que se ha proporcionado batalla (subcategoría: dinámica del juego). En ese sentido, el 70,0\% de los educandos están completamente de acuerdo que subieron del primer nivel al tercero y el $50,0 \%$ expresaron que alcanzaron el tercer nivel desde el segundo. Mientras que, lo referente subcategorías mecánicas de las actividades de retos y competencias matemáticas, el $83,3 \%$ recibieron información docente sobre su progreso. Entretanto que el $96,7 \%$ atendieron comentarios del docente sobre el éxito o el 
fracaso de sus progresos en dichos retos y competencias.

No obstante, la dinámica de juego descendió un $10.0 \%$ para algunos participantes en cuanto a la motivación, pues en la mecánica de juego un $13,3 \%$ estuvo en desacuerdo con la información recibida y otro 3,3\%, no respondió a los comentarios sobre los retos y competencias que hizo el docente. Aunque, el 93,3\% se olvidó del paso del tiempo mientras realizaba los retos de matemática en el sitio web. De allí, que la totalidad dijeran que disfrutaron de la dinámica del juego.

Por otra parte, se logró determinar que durante el proceso de gamificación de las matemáticas en la herramienta Web, el 93,3\% están completamente de acuerdo y bastante de acuerdo que el sitio web de gamificación generó entusiasmo y por eso se olvidó del paso del tiempo al realizar las actividades de matemática mientras navegaban por este sitio web. Por ello, el 100,0\% expresaron en estar de completamente de acuerdo y bastante de acuerdo con el disfrute de la dinámica del juego.

Los resultados obtenidos en los ítem del 1 al 9, caracteriza la alternancia de elementos dentro y fuera del juego, que ayudan a crear conciencia sobre los objetivos de aprendizaje entre la dinámica y mecánica del juego, pues, produce acción en el participante y promueve la resolución de problemas dentro de la mecánicas del juego (Kapp 2012), ya que en la (tabla 2), se evidencia que el estudiante recibió información durante el progreso de los retos y competencias (media=0,4 ; DT=1,0), junto a la facilidad de uso motiva realizar actividades de matemática (calificación promedio de 4,9, DT=0.3), y el disfrute de realizar problemas matemáticos con los elementos del juego (calificación promedio de 4,7, DT=0,5), motiva avanzar en los niveles y obtener los puntos de recompensas (media= $4,2, \mathrm{DT}=1,0)$.

De este modo, se logra transferir conocimientos a nuevas situaciones y habilidades en la signatura de matemática y el desarrollo de otras habilidades transferibles en la asignatura al asumir los retos y obtener recompensas en la gamificación, lo cual aumentó el compromiso por el logro debido a sus puntos y ranking en los distintos niveles (Ortegón, 2016).

Además, los elementos de la gamificación deberíanirseguidos deinformaciónocomentarios del docente sobre lo que se ha experimentado en la dinámica, mecánica y componentes del juego. Por tanto, la realimentación debe enfocarse en las habilidades de trabajo individual y en equipo, negociación y resolución de problemas matemáticos durante el juego, haciendo pensar en lo que se podría haber hecho de manera diferente mientras se juega aprendiendo. 


\subsection{Percepciones de los estudiantes de matemática sobre la realimentación y la evaluación formativa del docente en un ambiente gamificado}

Tabla 3. Apreciaciones promedio de los estudiantes de matemáticas a los efectos de utilizar la evaluación formativa.

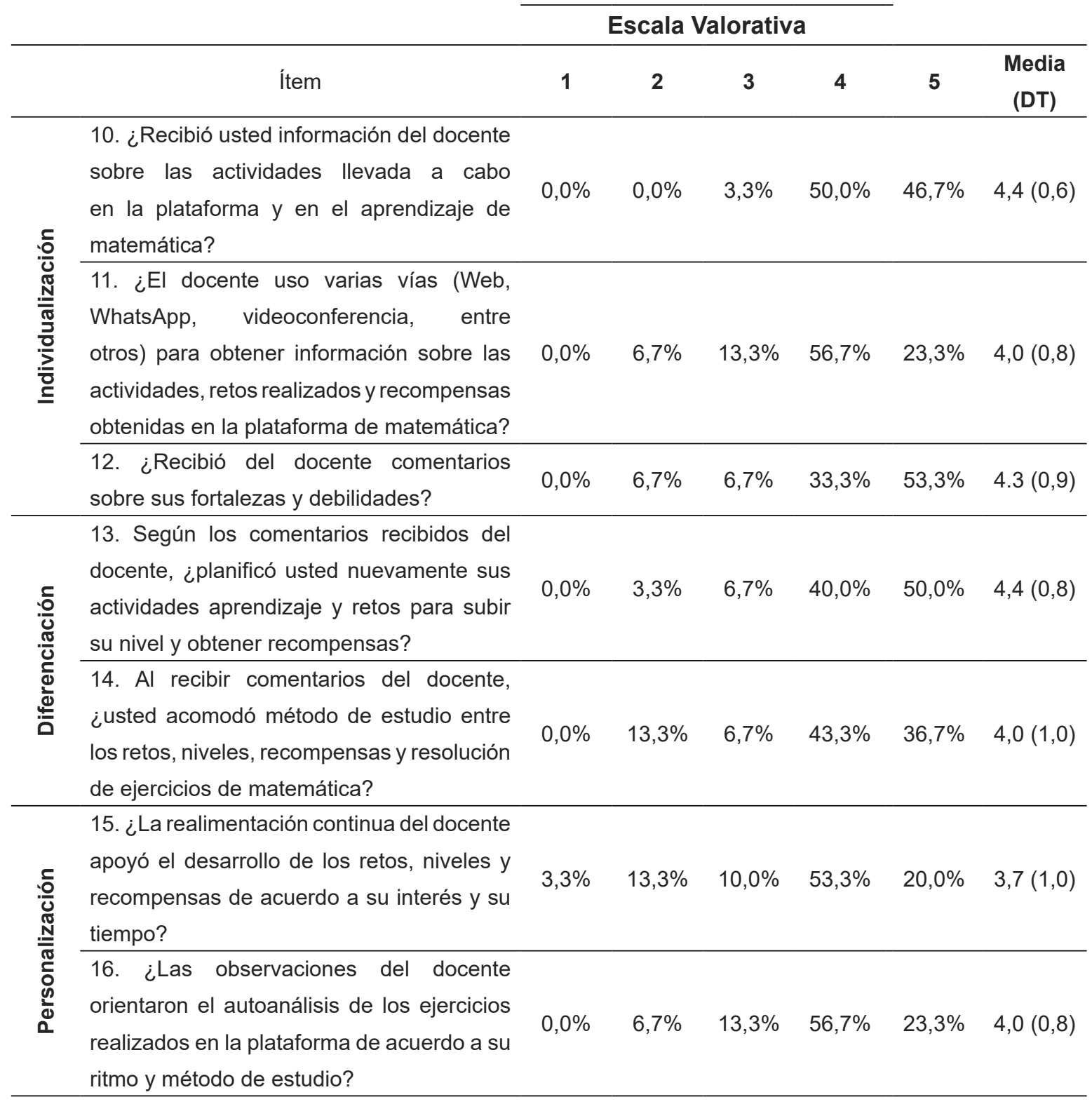

Escala evaluativa: 1. Totalmente en desacuerdo; 2. En desacuerdo; 3. Indiferente; 4. De acuerdo; 5.

Totalmente de acuerdo 
Los resultados mostraron (Tabla 3) que los estudiantes de matemáticas están bastante de acuerdo y completamente de acuerdo en recibir realimentación formativa de la docente llevada a cabo en la plataforma y en el aprendizaje de matemática $(96,7 \%)$, a través de varias vías (Web, WhatsApp, videoconferencia, entre otros) sobre las actividades, retos realizados y recompensas obtenidas en la plataforma de matemática $(80,0 \%)$ para fortalecer sus debilidades $(86,7 \%)$.

El $90,0 \%$ de los encuestados están bastante de acuerdo y completamente de acuerdo que luego de recibir comentarios del docente, definitivamente planificaron sus actividades aprendizaje y retos para subir su nivel y obtener recompensas. El 80,0\%, acomodaron el método de estudio entre los retos, niveles, recompensas y resolución de ejercicios de matemática. Donde el 83,3\% expresó que la realimentación continúa del docente apoyó el desarrollo de los retos, niveles y recompensas de acuerdo a su interés y su tiempo. Esto se debe a que las observaciones del docente orientaron el autoanálisis de los ejercicios realizados en la plataforma de acuerdo a su ritmo y método de estudio $(80,0 \%)$.

Al analizar los ítems del 10 al 16, se constató que la evaluación formativa en los estudiantes, permite y da la oportunidad de asumir la responsabilidad de su propio ritmo de aprendizaje (individualización), ya que el docente apoya el desarrollo de las competencias de matemática y ayuda a comprender el contenido de los objetivos de aprendizaje, así como a vincular los objetivos de aprendizaje con la práctica gamificada para que ajusten los métodos de aprendizajes (diferenciación).

Con lo que se da una personalización del aprendizaje al conocer los conocimientos y habilidades previos de los estudiantes, pues, recopila información sobre los supuestos matemáticos y habilidades prácticas de los estudiantes. Lo que concuerda con Santiago y Bergmann (2018) que el docente tiene la posibilidad mediante la evaluación formativa de ajustar el proceso paulatinamente de los estudiantes a través de la individualización, diferenciación y personalización de la evaluación.

Aunque, las opiniones de los estudiantes demostraron significancias en las medias obtenidas, porque la realimentación en evaluación formativa tienen efectos positivos, lo que coincide con Welbers et al. (2017) quienes relacionan que la retroalimentación posee un resultado efectivo en la participación de los jugadores, ya que se fundamenta en recopilación información sobre las actividades a realizar (media $=4,4$ y DT $=0,6$ ) para adaptarse y planificar nuevamente sus actividades (media=4,4; DT= $0,8)$, pero con las consideraciones del docente de acuerdo a ritmo y método de estudio de cada estudiante (media $=4,0 ; \mathrm{DT}=0,8$ ).

Por tanto, la realimentación formativa involucra indagación participada al educando con la finalidad de transformar su actuación personalizadas con la intención de optimizar el aprendizaje mediante el autoanálisis de lo que vienen haciendo. Lo que concuerda con el planteamiento de Pérez, Enrique, Carbó y González (2017) que destacan que lo formativo es un control dentro de la evaluación que contribuye al avance del estudiante en correspondencia con las observancias del docente. Este es un tema importante para estudios futuros, que podrían centrarse en si la cantidad y el tipo de personalización marca la diferencia

La comparación de los valores medios de los grupos reveló que utilizar la evaluación formativa en una herramienta Web de la asignatura de matemática en un ambiente no lúdico y gamificado no dependen del docente $(p>0,05)$ sino de los métodos de realimentación utilizados 
en dar respuestas a las inquietudes y solicitudes presentadas por los estudiantes.

\subsection{Métodos de evaluación y métodos de retroalimentación utilizados en la herramienta Web gamificada}

Luego de hacer un análisis de la información encontrada en la Web de Retomates (Grupo de gestión en la zona de profes) los métodos para verificar los niveles de comprensión de las matemáticas (evaluación) más utilizados por los estudiantes en la herramienta Web gamificada son los retos individuales $(93,3 \%)$ y el menos usado es el autoanálisis $(46,7 \%)$ ver tabla 4.

Tabla 4. Métodos de evaluación gamificada y métodos de retroalimentación

\begin{tabular}{|l|c|c|}
\hline \multicolumn{1}{|c|}{ Método } & $\mathbf{f i}$ & $\%$ \\
\hline Retos individuales & 28 & $93,3 \%$ \\
\hline Batallas en grupo & 22 & $73,3 \%$ \\
\hline Trivia en grupo & 20 & $66,7 \%$ \\
\hline Resolución de ejercicios prácticos & 25 & $83,3 \%$ \\
\hline Entrevistas con el docente & 24 & $80,0 \%$ \\
\hline Observación del docente & 27 & $90,0 \%$ \\
\hline $\begin{array}{l}\text { Información y comentarios del docente al } \\
\text { estudiante }\end{array}$ & 28 & $93,3 \%$ \\
\hline Comentarios de estudiante al docente & 23 & $76,7 \%$ \\
\hline Comentarios de estudiante a estudiante & 25 & $83,3 \%$ \\
\hline Autoanálisis del estudiante & 14 & $46,7 \%$ \\
\hline
\end{tabular}

En la herramienta Web de matemáticas, tanto en los eventos grupales de las batallas (73,3\%) como en las trivias $(66,7 \%)$ y la resolución de ejercicios prácticos $(83,3 \%)$ también utilizan las entrevistas con los docentes (80,0\%). El 90,0\% de los encuestados acogen como positiva utilización de la observación como método de evaluación formativo en sus actividades gamificadas para recibir realimentación del docente, el cual es el más popular entre los encuestados $(93,3 \%)$, seguido de la revisión de pares $(83,3 \%)$. Es decir, más de del $80,0 \%$ de los estudiantes analizan su propio trabajo de aprendizaje, así como de la realimentación de estudiante a docente (76,7\%).

A continuación, se presenta el análisis de las categorías de acuerdo a la Observación directa. 
Tabla 6. Observación directa en clase

\begin{tabular}{|c|c|}
\hline Catec & Observación directa \\
\hline Motivación & $\begin{array}{l}\text { Se observó que en la experiencia en la herramienta gamificada, los participantes realizaban } \\
\text { preguntas a sus pares y al docente, tanto en la comunicación directa de la plataforma como } \\
\text { por el grupo de comunicación de WhatsApp, así como videoconferencia por Meet. } \\
\text { Los comentarios de profesor a estudiante, así como de estudiante a docente y estudiante- } \\
\text { estudiante no eran tomados con negatividad, pues el disfrute por el juego elimina cualquier } \\
\text { nivel de estrés por resolver los retos y batallas planteados durante las actividades gamificada. } \\
\text { De igual manera, se evidenció una inmersión de los estudiantes en la resolución de los retos y } \\
\text { batallas en la plataforma de matemática. De allí, que la mayoría de los estudiantes estuvieron } \\
\text { siempre motivados en la realización de las actividades ya que iban ganando confianza por si } \\
\text { solos al repetir una y otra vez los retos. }\end{array}$ \\
\hline Gamificación & $\begin{array}{l}\text { La gamificación adquiere una variedad de significados en el proceso de enseñanza } \\
\text { aprendizaje según el estudiante (jugador), la situación del juego (gamificación) y el control } \\
\text { formativo que ejerce el docente. } \\
\text { Existe un número ilimitado de combinaciones entre la dinámica, la mecánica del juego, los } \\
\text { componentes de la misma y las retroalimentaciones que el docente pude dar (información y } \\
\text { comentarios), ya que en un principio un nutrido grupo de estudiante recurrieron al docente } \\
\text { para comprender las actividades gamificada. } \\
\text { Se observó la importancia del juego para el educador, el control formativo del juego y sus } \\
\text { orientaciones en la mecánica del mismo. } \\
\text { El enfoque de control formativo y gamificación está relacionado entre sí en la herramienta } \\
\text { Web de matemática, de acuerdo a los avances del estudiante, los retos, batallas o trivias y } \\
\text { el apoyo del docente o un amigo para continuar con aspectos conceptuales y resolución de } \\
\text { problemas matemáticos. Lo cual obtendría una realimentación de una zona proximal en su } \\
\text { proceso de aprendizaje. } \\
\text { Esta interpretación entre los hallazgos es interesante porque fortalece la categoría de evaluar } \\
\text { formativamente las actividades que realiza el jugador (estudiante). }\end{array}$ \\
\hline $\begin{array}{l}\text { Evaluación } \\
\text { formativa y } \\
\text { realimentación }\end{array}$ & $\begin{array}{l}\text { Se observó que las actividades de digitales recopilan y producen información sobre las } \\
\text { actividades gamificada realizadas. Primero, brindan apoyo entre pares y segundo apoya } \\
\text { al docente en el control que puede ejercer sobre el grupo para retroalimentar a sus } \\
\text { estudiantes formativamente. } \\
\text { También, se constató que se presentan diversas limitaciones con respecto al desarrollo de } \\
\text { evaluación formativa personalizada en los elementos de gamificación para la herramienta } \\
\text { digital. Puesto que, el docente presentó limitaciones técnicas en dichas herramientas } \\
\text { digital, independientemente de que esta última proporcione información sobre lo que hacen } \\
\text { los estudiantes, pero no sobre los problemas que enfrenta con la mecánica, dinámica o } \\
\text { componentes del juego en sus actividades académicas digitales, lo que podría dificultar la } \\
\text { evaluación formativa individualizada. } \\
\text { De igual manera, se contrastó que los estudiantes al evaluar los métodos de aprendizaje } \\
\text { en un proceso constantemente de entrevistas, pregunta-respuesta y observaciones toma } \\
\text { la iniciativa para interactuar con la dinámica y mecánica del juego en la resolución de } \\
\text { problemas matemático basados en las informaciones y comentarios obtenidas. }\end{array}$ \\
\hline
\end{tabular}




\subsection{Pasos hacia una evaluación formativa en la gamificación de la asignatura de matemática}

Al evaluar la acción ejecutada y comparar los resultados de la presente indagación junto a la literatura sobre el tema, nos lleva a subrayar que la transición de la apreciación de control en una herramienta digital a la evaluación formativa en contexto gamificado de la asignatura de matemática no es sencilla, requiere un cierto número de condiciones propicias para efectos formativos que delimitan las actuaciones que se desea lograr con respecto a las actividades sin olvidar la diversión en la implementación adecuada de la herramienta (Werbach y Hunter, 2012).

La experiencia llevada a cabo ha puesto de manifiesto la ambigüedad del posicionamiento de la motivación en el juego entre la gamificación, la herramienta Web gamificada y la evaluación formativa. La herramienta digital gamificada (retomates), al precipitar un pasaje de lo virtual a una forma de explicitación con respecto a la enseñanza (gestión de grupo de zona de profes) y la visibilidad de las evaluaciones de los estudiantes sobre ella (retos, batallas, trivia), lleva a cuestionar las orientaciones de control formativo del docente en la dinámica, mecánica y componentes de la gamificación, así como sus interacciones.

Porque la dinámica de juego en las actividades de matemática gamificadas puede desarrollar experiencias emocionales relacionadas con la motivación (Ortiz-Colón, Jordán y Agreda, 2018, p.3) sin contar de los comentarios de los docentes, por la facilidad de uso de la plataforma por razón de juegos interactivos en una web (Perea, 2016).

Eso, hace posible la participación de todos los estudiantes; su adaptabilidad en la dinámica y mecánica de juego, ya que la misma se basan en el éxito y el fracaso, y los estudiantes al practicar pueden lidiar con los errores de manera segura frente a ellos, pues la mecánica y componentes del juego lo establecen de esa manera. Pareciera que no se hace propicia para una evaluación contextualizada y evolutiva que se presenta en la herramienta gamificada utilizando técnicas de evaluación informal, semiformal y formal (González, Hernández y Hernández, 2007) que muchas veces no se llevan a cabo.

Al mismo tiempo, la dinámica de las actividades gamificadas fomentan la perseverancia ofreciendo nuevos intentos hasta que el jugador (estudiante) se adelanta y es recompensado por intentarlo. Por tanto, se deduce que dinámica las actividades gamificadas es un factor motivador en el uso de la herramienta digital de matemática por parte de los estudiantes, pero se considera que es necesario la personalización, la diferenciación y la individualización en el proceso de evaluación formativa (Santiago y Bergmann, 2018).

De este modo, para que el aprendizaje de las matemáticas no se quede solo en una competencia de retos, trivias y batallas, se requiere de la evaluación formativa en el ambiente no lúdico, que ofrece realimentación por parte del docente a la experiencia del estudiante, con el fin de dicha experiencia sea más depurada, mejorando la integración de los educandos con los elementos gamificados dentro de la plataforma digital a través de un apoyo pedagógico, pues se observó que en las batallas, los estudiantes solo disfrutaban de la competencia que se generaba con sus pares, porque más de una de las terceras partes de los estudiantes consideraron que sería útil contar con la ayuda del docente para dilucidar la información recibida, por ello, un sistema de apoyo educativo (evaluación formativa) les sería beneficioso a la gamificación de las matemáticas en ambientes no lúdicos. 
De allí, que las sinergias entre la gamificación y evaluación formativa, ligadas a los compromisos de los actores de aprendizaje, permitieron que los estudiantes pasaran de un nivel a otro en la comprensión de los conceptos matemáticos establecidos, así como en la dinámica y mecánica de juego de la asignatura dentro del contexto digital en sus distintos componentes (niveles, recompensas, insignias, entre otros), donde el docente apoyó el desarrollo del estudiante en su potencial para mejorar el aprendizaje (Martínez Rizo, 2012).

Por lo tanto, la gamificación en ambientes no lúdicos junto a la evaluación formativa contribuyó para que los estudiantes alcanzaran los niveles de comprensión de matemática planteados tras segmentos frecuentes de realimentación, lo que se da respuesta a la hipótesis planteada, aunque la misma se deben establecer varias condiciones tanto a nivel pedagógicos como sociocognitivo, a saber, un control formativo claramente diferenciado de un objetivo de control sumativo de calificaciones y una responsabilidad en la gamificación (Ortegón, 2016) de los actores en un proceso de reflexividad colectiva.

\section{CONCLUSIONES}

Los resultados de este trabajo permiten obtener una visión general de la implementación de la evaluación formativa y la gamificación en la asignatura de matemática en el contexto de una herramienta digital. Primero, la evaluación formativa es un método cualitativo de evaluación que hace la enseñanza más efectiva a través del asesoramiento y la realimentación incide en el mejoramiento del rendimiento del aprendizaje, ya que determina el nivel actual de los estudiantes al identificar conocimientos y habilidades previos. Seguidamente, involucra a los estudiantes en la planificación de la evaluación porque proporciona información y acotaciones sobre sus fortalezas y debilidades a los estudiantes. Donde el punto de vista primordial sería la evaluación de apoyo al estudiante para obtener un logro o recompensa dentro del juego.

Al mismo tiempo, analiza la propia enseñanza en función de la percepción de los estudiantes motivados en dicho contexto de juego para realizar realimentaciones acordes a las asignaciones propuestas, tiempo y ritmos de cada uno, así como de la dinámica y mecánica de los objetivos gamificados con respecto a la evaluación con el uso de diferentes métodos formativos.

Se concluye que cuanto más aumenta la gamificación en sus elementos y la evaluación formativa en el contexto digital más cambia significativamente el aprendizaje de las matemática, puesto que, los elementos que utiliza la gamificación en ambientes digitalizado crea eventos, donde la realidad se modela bajo la realimentación del docente que básicamente presume revelar los objetivos y juicios de evaluación, aparte de, proveer visibilidad a la voz de las perspectivas de los estudiantes sobre la dinámica, mecánica y componentes del juego, así como adaptación con respecto a los objetivos gamificados de la asignatura.

\section{REFERENCIAS}

Araya, V, Alfaro, M. \& Andonegui, M. (2007). Constructivismo: orígenes y perspectivas. Laurus, 13(24),76-92. https://www.redalyc. org/articulo.oa?id=761/76111485004

Aristizábal, JH; Colorado, H \& Gutiérrez, H. (2016) El juego como una estrategia didáctica para desarrollar el pensamiento numérico en las cuatro operaciones básicas. Sophia 12 (1): 117-125. http://www.scielo.org.co/pdf/sph/ v12n1/v12n1a08.pdf

Brenes, F. (2006). Evaluación diagnóstica, formativa y sumativa de los aprendizajes. Costa Rica: EUNED.

Casallas, Ly Maheca, H. (2019) Uso de estrategia 
didáctica apoyada en la gamificación para el desarrollo de habilidades en el planteamiento y resolución de problemas aritméticos, en instituciones educativas rurales. Universidad Cooperativa de Colombia.

Castillo, S. (2008). Propuesta pedagógica basada en el constructivismo para el uso óptimo de las TIC en la enseñanza y el aprendizaje de la matemática. Revista latinoamericana de investigación en matemática educativa, 11(2), 171-194.http://www.scielo.org.mx/ scielo.php?script $=$ sci_arttext\&pid=S1665$24362008000200002 \&$ Ing=es\&tlng=es

Castro, F. S. de. (2016). A gamification framework as a collaboration motivator for software development teams. Pontifícia Universidade Católica do Rio Grande do Sul. https://repositorio.pucrs.br/dspace/ handle/10923/8570

Cobo, C. y Pardo, H. (2007). Planeta Web 2.0. Inteligencia colectiva o medios fast food. (Grup de Recerca. d'Interaccions Digitals, Universitat de Vic. Flacso México.). Barcelona/México DF.

Colomo, E, Sánchez, S., Ruiz, J. y Sánchez, J. (2020). Percepción docente sobre la gamificación de la evaluación en la asignatura de Historia en educación secundaria. Información Tecnológica - Vol. $31 \mathrm{~N}^{\circ} 4$ http://dx.doi.org/10.4067/S071807642020000400233

Creswell, J. W. (2012). Educational research. Planning, conducting and evaluating quantitative and qualitative research. ( $4^{\mathrm{a}}$ ed.). USA: Pearson.

Csikszentmihalyi, M. (1990). De flujo: la psicología de la experiencia óptima. Nueva York, Harper \& Row

Dicheva, D., Dichev C., Agre G., \& Angelova G. (2015). Gamification in Education: A Systematic Mapping Study. Educational Technology \& Society, 18 (3), 75-88. https://www.jstor.org/stable/ jeductechsoci.18.3.75?seq=1\#metadata info_tab_contents

Erenli, K. (2013). The Impact of Gamification - Recommending Education Scenarios. iJET - Volume 8, Special Issue 1: https:// online-journals.org/index.php/i-jet/article/ view/2320

Fernández, M. (2017). Evaluación y aprendizaje. MarcoELE: Revista de Didáctica Español Lengua Extranjera, 24, 3. https://dialnet.unirioja.es/servlet/ articulo?codigo $=6145807$

Ferguson, R., Brasher, A., Clow, D., Cooper, A., Hillaire, G., Mittelmeier, J., Rienties, B., Ullmann, T., Vuorikari, R. (2016). Research Evidence on the Use of Learning Analytics - Implications for Education Policy. R. Vuorikari, J. Castaño Muñoz (Eds.). Joint Research Centre Science for Policy Report; EUR 28294 EN; doi: 10.2791/955210.

García-Quintero, C., \& Villamizar Suárez, G. (2017). Análisis fenomenológico de la conciencia del docente a partir de sus prácticas evaluativas. Revista Perspectivas, 2(2), 49-59. https://doi. org/10.22463/25909215.1313

González, M., Hernández, A. \& Hernández, A. (2007). Elconstructivismoenlaevaluaciónde los aprendizajes del álgebra lineal. Educere, 11(36), 123-135. http://ve.scielo.org/ scielo.php?script=sci_arttext\&pid=S131649102007000100016\&lng=es\&tIng=es

Holguín, F.; Holguín, E. y García, N. (2020). Gamificación de la enseñanza de las matemáticas: una revisión sistemática. Telos: revista de Estudios Interdisciplinarios en Ciencias Sociales, 22 (1), pp.62-75. DOI: www.doi.org/10.36390/telos221.05

Kapp, M. (2012) The mification of learning and instruction: Game-based Methods and strategies for Training and education. John While\& Sons.

López-García, J. D., \& Gutiérrez-Niño, D. (2018). Efecto del uso de la herramienta "realidad aumentada" en el rendimiento académico de estudiantes de Educación Básica. 
Revista Perspectivas, 3(1), 6-12. https:// doi.org/10.22463/25909215.1464

Lleixà, T.; Gros, B.; Mauri, T.; Medina, J.L (eds.) (2018) Educación 2018-2020. Retos, tendencias y compromisos. Barcelona: IREUB. http://www.ub.edu/grop/wp-content/ uploads/2018/07/2018-cap-Lleixa-perezbusca-IRE-UB_CASTscreen-1.pdf

Martínez, M. (2004). Ciencia y arte en la metodología cualitativa. Editorial Trillas. S. A. de C. V.

Martínez, G. (2017) Tecnologías y nuevas tendencias en educación: aprender jugando. El caso de Kahoot. Opción, vol. 33, núm. 83, https://produccioncientificaluz. org/index.php/opcion/article/view/23116

Martínez Rizo, F. (2012). La evaluación formativa del aprendizaje en el aula en la bibliografía en inglés y francés: Revisión de literatura. Revista mexicana de investigación educativa, 17(54), 849875. http://www.scielo.org.mx/scielo. php? script $=$ sci_arttext $\&$ pid $=$ S1405$66662012000300008 \&$ lng=es\&tlng=es.

Ministerio de Educación Nacional (MEN, 2009). Decreto No. 1290. Reglamento de la evaluación del aprendizaje y promoción de los estudiantes de los niveles de educación básica y media.

Mora, C. (2003). Estrategias para el aprendizaje y la enseñanza de las matemáticas. Revista de Pedagogía, 24(70), 181272. Recuperado en 14 de febrero de 2021, de http://ve.scielo.org/scielo. php?script $=$ sci_arttext $\&$ pid $=$ S079897922003000200002\&lng=es\&tIng=es

Ortegón, M. (2016). Gamificación de las matemáticas en la enseñanza del valor posicional de cantidades. Trabajo final de Master. Universidad Internacional de La Rioja. https://reunir.unir.net/ handle/123456789/4704

Ortiz-Colón, A., Jordán, J. \& Agredal, M. (2018). Gamificación en educación: una panorámica sobre el estado de la cuestión.
Educação e Pesquisa, 44, e173773. Epub April 23, 2018.https://doi.org/10.1590/ s1678-4634201844173773

Penagos, M., Mariño, L. F., \& Hernández, R. V. (2017). Pensamiento matemático elemental y avanzado como actividad humana en permanente evolución. Revista Perspectivas, 2(1), 105-116. https://doi. org/10.22463/25909215.1289

Perea, D. (2016). Retomates, juegos para aprender en clase. XVI Congreso de enseñanza y aprendizaje de las matemáticas. Matemáticas, ni más ni menos. Sociedad Andaluza de Educación Matemática "THALES". p.396-412. https:// thales.cica.es/xviceam/actas/pdf/actas.pdf

Pérez, M., Enrique, J., Carbó, J. \& González, M. (2017). Laevaluación formativa enel proceso enseñanza aprendizaje. EDUMECENTRO, 9(3), 263-283. http://scielo.sld.cu/scielo. php?script $=$ sci_arttext $\&$ pid $=$ S2077 28742017000300017\&lng=es\&tlng=es

Restrepo, B. (2004). La investigación-acción educativa y la construcción de saber pedagógico. Educación y Educadores, (7), 45-55. https://educacionyeducadores. unisabana.edu.co/index.php/eye/article/ view/548

Rodríguez, G. y lbarra, M. (2013). Autoevaluación, evaluación entre iguales y coevaluación: conceptualización y práctica en las universidades españolas. Revista de Investigación en Educación, 11(2), 198210. https://idus.us.es/handle/11441/68957 Rojas, Y., Beleño, N., \& Valbuena, S. (2015). Aplicación de las TIC en el aprendizaje de las matemáticas. Revista MATUA 2(1), http://investigaciones.uniatlantico. edu.co/revistas/index.php/MATUA/article/ view/1347

Ropero, S. (2018) Aplicación de una metodología gamificada para la mejora de una unidad didáctica en formación profesional. Universidad Internacional 
de La Rioja. https://reunir.unir.net/ handle/123456789/7020

Sánchez, J. (2018). La gamificación a través de la plataforma Smartick para mejorar el rendimiento académico en matemáticas en estudiantes de la I.E.D. Tercera Mixta de Fundación - Magdalena. https://repositorio. cuc.edu.co/handle/11323/67

Salen, K. y Zimmenrman, E. (2003). Rules of play - game design fundamentals. Cambrige: MIT Pres

Santiago, R. \& Bergmann, J. (2018) Aprender al revés. Flipped learning 3.0 y metodologías activas en el aula. PAIDÓS Educación. Barcelona, España

Siemens, G. y Baker, R. (2012). Learning analytics and educational data mining: towards communication and collaboration. In 2nd International Conference on Learning Analytics and Knowledge. Congress held at Vancouver, BC, Canada. http://goo.gl/ xSXhWj.

Torres, A., Romero, L. y Pérez, A. (2017). Ludificación y sus posibilidades en el entorno de blended learning: revisión documental. RIED. Revista Iberoamericana de Educación a Distancia, vol. 21, núm. 1, DOI: https://doi.org/10.5944/ ried.21.1.18792

Vilches, D. (2014). Juegos Serios, evaluación de tecnologías y ámbitos de aplicación. Tesis de especialización. Universidad Nacional de La Plata http://sedici.unlp. edu.ar/bitstream/handle/10915/40330/ Documento_completo.pdf?sequence $=1$

Villanueva, G. \& Casas, M. (2010). E-competencias: nuevas habilidades del estudiante en la era de la educación, la globalidad y la generación del conocimiento. Signo y Pensamiento, XXIX (56),124138. https://www.redalyc.org/articulo. oa? $\mathrm{id}=860 / 86019348008$

Welbers, K.; Konijn, E.; Burgers, C. Bij de Vaate, A. Eden, A, \& Brugman, C. (2019). Gamification as a tool for engaging student learning: A field experiment with a gamified app. E-Learning and Digital Media. Volume: 16 issue: 2, page(s): 92-109. https:// journals.sagepub.com/toc/ldm/16/2

Werbach, K., \& Hunter, D. (2012). For the win: How game thinking can revolutionize your business. Harrisburg: Wharton Digital Press. 